\title{
Research on Innovation of Health Qigong Routine Competition System Under the Vision of Supply Side Reform*
}

\author{
Wen Wang \\ The Physical Education Department \\ Jilin Institute of Chemical Technology \\ Jilin, China 132022 \\ Kyungnam Univeristy \\ Changwon-Si, Republic of Korea 51767
}

\begin{abstract}
With the acceleration of the supply-side structural reform and the development of the internationalization path, the fitness Qigong routine competition system cannot meet the diverse needs of the masses in terms of project setting, content innovation, time and place. The reform of the Health Qigong Sports Competition System has a strong driving value for constructing the context of leisure sports culture and clarifying the entanglement of the traditional cultural system. Under the supply-side reform perspective, the location and time of the fitness Qigong routine competition, the establishment of the competition system innovation project, the innovation and development of the competition method, and the competition model should be correspondingly innovated.
\end{abstract}

Keywords-supply side reform; fitness qigong; routine competition; competition system innovation

\section{INTRODUCTION}

Qigong is one of the traditional Chinese national sports culture projects. The Qigong movement first appeared in the Spring and Autumn Period and the Warring States period, and gradually formed in the Han Dynasty. From the perspective of historical origin, Qigong movement is developed and interpreted from the martial arts movement and is a branch of martial arts. Modern Qigong movement started in the 1950s. After the government's policy support and promotion, it has gradually become a routine project in the national cultural sports competition system. A large number of national championships, international invitational tournaments, minority sports games, and peasant sports games have been held nationwide. At present, Yi Jinjing, Wu Qin Xi, Liu Zi Yan, and Ba Duan Jin have gone out of the country and held many international Qigong Invitational Tournaments. [1] However, with the acceleration of the

*Fund Project: Youth Project of Humanities and Social Science Fund of the Ministry of Education (Project No.: 18YJC890044); Scientific and Social Science and Technology Department of Jilin Province "13th FiveYear" Scientific Research Project (Project No.: JJKH20190839SK); Jilin Higher Education Society 2017 Higher Education Research Project (Project No.: JGJX2017D205); 2016 Jilin University of Chemical Technology major research. supply-side structural reform and the development of the internationalization path, the Health Qigong Routine Competition System cannot meet the diverse needs of the masses in terms of project setting, content innovation, time and place.

\section{THE NECESSITY AND CULTURAL ORIENTATION OF THE REFORM OF HEALTH QIGONG ROUTINE COMPETITION SYSTEM DEFINITION OF RELATED CONCEPTS}

The characteristics of China's Health Qigong movement are obvious, and the mass foundation is profound, which lays a solid foundation for the reform of the Health Qigong routine competition system. The reform of the Health Qigong Sports Competition System has a strong driving value for constructing the context of leisure sports culture and clarifying the entanglement of the traditional cultural system.

\section{A. The Characteristics of Qigong Movement Are Obvious, and the Competition System Has Been Basically Formed}

On the one hand, in China, the Qigong movement has a profound mass base and is loved by the masses because of its own national fitness value, competitive nature and artistic characteristics. At present, the four exercises of Yi Jin Jing, Wu Qin Xi, Liu Zi Yi and Ba Duan Jin are mainly divided into three modes. The first one is a highly technical and ornamental project in the modern sense. It inherits the essence of traditional Qigong movement and is innovative and has a strong appreciation value. The second is competitive Qigong. The competitive Qigong is mainly based on the basic skills of Qigong, which combines the content of confrontation and makes the competitive characteristics of the sport manifest. The game includes multiple modes such as single player, doubles and team competition. This type of Qigong competition was launched in the peasant games and national sports games held in China. The third is the popular Qigong, which is the movement of the people in their daily lives to entertain themselves. The type of the project is not limited by time and venue. The gameplay is diverse, flexible and has good fitness attributes. 
On the other hand, the characteristics of Qigong exercise are obvious; in general, it is scientific, fitness, entertainment, competitive and many other characteristics. Scientific means that the formation of this movement conforms to the characteristics of human body mechanics, can effectively adapt to various living and production environments, and has a strong promotion effect on the physical and mental development of the human body and physical fitness. Its technical operation can be easily changed to difficult, science Training, step by step. Fitness means that the exercise not only exercises the neck, shoulders and legs of the human body, but also helps the body function of the knees, hips, ankles, etc. to enhance the flexibility of the human body and enhance the individual's constitution. [2] Entertaining means that the project is highly entertaining and is not affected by age, gender, occupation, weather, venue, etc. As long as it is a normal group, it can actively participate. Competitiveness mainly refers to the use of basic skills in competitive Qigong, which combines the content of competitive confrontation and makes the competitive characteristics of the sport manifest. The game includes multiple modes such as single player, doubles and team competition.

\section{B. Constructing a Vein of Leisure Sports Culture and Clarifying the Entanglement of the Traditional Cultural System}

The history of the development of human civilization is not a history, but full of repetition and twists and turns. The development of leisure sports culture is also true. In a certain historical stage, the national traditional sports culture, as a subculture, carries the change of values and challenges the change of mainstream values. [3] However, the development of history requires that sports culture should be gradually transformed by mainstream culture, and form a cultural phenomenon that mainstream culture can accept. In this context, the Qigong movement of the national traditional sports project is deeply imprinted with the Confucian cultural imprint, and is regulated and restrained. Therefore, from the inheritance and promotion of the Qigong movement of the national traditional sports project into the campus, it's possible to construct the context and characteristics of the ancient leisure sports culture and its integration with the traditional cultural system. Based on the analysis of Qigong development and the setting of competition system, it can effectively understand the norm and formation of ancient leisure sports culture. The standardization and formation of ancient Chinese leisure sports culture are mostly inseparable from the changes in economic customs. Based on military political needs, they are linked to the social operation mechanism. First, the origin of Qigong is inseparable from the changes in economic customs. Sports and leisure projects such as Qigong have no material basis. Similar to qigong games and entertainment activities, it can show the instinct of the Chinese people, so that it can spread better. Second, the development of Qigong based on military political needs highlights the characteristics of the project. Leisure sports have the characteristics of diffusion, and the diffusion between different systems and the diffusion of different structures in the same culture form a unique cultural phenomenon. In the Han Dynasty, with the economic and social development, Qigong gradually merged with music and became part of the court performance. During the Southern and Northern Dynasties, the qigong culture as a form of performance was further spread under the foil of sports and leisure roles. The third is to explore the linkage of the social operation mechanism behind the prosperity of Qigong, which can provide a basis for the current sports improvement. With the introduction of foreign and minority cultures, the cultural environment is more relaxed and harmonious, and the sports and leisure projects are more abundant. The entertainment and appreciation of the Qigong movement itself is also highlighted. The Qigong movement has formed a unique characteristic, the leisure sports culture has grown, and it has produced a positive interaction with the social operation.

\section{ANALYSIS OF THE CURRENT SITUATION OF THE CURRENT FITNESS QIGONG ROUTINE COMPETITION SYSTEM}

At present, the development of China's Health Qigong routine competition is better, but there are still some shortcomings in the time and place, method and project setting.

\section{A. Venue Location and Time Status}

The schedule of the competition includes the preparation time, the competition time and the organization management time. The duration of the competition is more than one week. The registration and meeting schedule is about 1-3 days. The competition time is about 4 days. The meeting time is the same as the competition time. Judging from the venue of the event, the venue of the event has a fixed location to hold a single type of project, as well as a series of tournaments, championships, and challenges held in various parts of the country. [4] There are certain problems in the time and place setting of the game. In the performance, some coaches and athletes hope that the game time is fixed, do not arbitrarily change the time, athletes and coaches can arrange training according to the specific schedule, improve the effectiveness of the training cycle. In addition, the competition for different regional events can effectively enhance the visibility of the region, but also to see the use of the network and modern information communication technology can achieve the corresponding effect. Therefore, the setting of different competition zones will increase the expenditure of the participating teams. The overall promotion effect and benefits are not great, and the commercial value cannot be effectively realized. Therefore, the competition time is fixed and the competition and athlete training can be a good competition, effectively reducing the cost and burden of the event.

\section{B. Problem with the Setting of the Competition Project}

Studies have shown that in the participating teams of Health Qigong, the competition project facilities, the number of participants and the content of the project are different, resulting in a large difference. The international competition is dominated by one-way, and the domestic competition is dominated by the all-around team competition. The setting of the event project is similar to the Taiji Zen Taijiquan 
competition, which has increased the individual all-around team competition, the Taijiquan project, and added a pair of pairs. Both the National Games project and the development of the two-person project have contributed to the development of competitive sports. Therefore, the Chinese fitness qigong routine's match mode can effectively match the international level, in order to improve its overall level. Judging from the actual effect of project setting, different sports teams attach great importance to collective projects and demonstrate overall strength through group cooperation. Group project setup requires at least 7 athletes to participate, and the group project makes the difficulty lower, and there is a drawback in reducing the number of athletes practicing individual projects. Overall, the guiding nature of project setup is very important. Only a relatively stable project and setting can promote the development of the overall competitive sports and have a strong guiding significance for the strategic development of traditional sports in China. It can effectively innovate and protect the competition project, activate the development of the event, and promote the rapid progress of competitive sports.

\section{Insufficient Entry Method and Competition Method}

The method of participation refers to the organizer's determination based on the team and the range of athletes. In order to protect athletes and ages, they are generally set at 16 years of age or older. At present, athletes in China generally demand to increase the number of places in the competition, so that more athletes can be qualified, show strength, expand the team, and promote the scale of the event. In the competition method, the arrangement of the competition sessions is carried out on the venues in the gymnasium. No matter the audience, the athletes, the referees and the coaches gather here, the energy is large, the time is too long, and the athletes participate in the competition. It has caused the difficulty of restoring physical strength, unable to effectively display the high level of exercise, and also produced some sports mistakes, which affected the development of the key body Qigong routine.

\section{Defects and Deficiencies in the Rules of the Competition}

The routine structure of Health Qigong in China has been greatly developed, but there is still no clear content structure in the competitive sports and fitness routines in the specific routine framework. Lack of detailed regulations, athletes and coaches also have more room to play. During the competition, the athletes combined the routines with the simple movements, and there was no definition and regulation in the rules, which caused the competitive sports routines to be completely dispelled. For example, in the development of Taijiquan athletic routines, athletes need to report the name of the routine, starting from the basic movements. In order to facilitate the movement, some athletes omit some of the movements, destroying the typical and normative routines, and weakening the skill. The martial arts routines transform like gymnastics.

\section{InNOVATION OF HEALTH QIGONG Routine COMPETITION SYSTEM UNDER THE VISION OF SUPPLY SIDE REFORM}

Under the supply-side reform perspective, the location and time of the Health Qigong routine competition, the innovative project setting of the competition system, the innovation and development of the competition method, and the competition model should be correspondingly innovated.

\section{A. Improving the Venue and Time of the Competition}

Under the supply-side reform, the needs of athletes and coaches must be taken seriously. The fixed competition time is conducive to the regularity of the event, which is convenient for coaches and athletes to grasp the exercise cycle and training routines to ensure full play of economic ability. Therefore, the location of the competition can be adjusted organically to increase the diversity of the event, and the fixed location can play a good publicity effect. Exploring the venues of the cycling system will facilitate the promotion of the entire event in a larger scale, and effectively set and rationally set according to the purpose and diversity of the event.

\section{B. Improving the Competition System Innovation Project Settings}

Under the supply-side reform, our national Health Qigong event has been shrinking in the setting of competitions. The Qigong Routine belongs to the skilloriented bodybuilding project. The intensity of the event and the content of the competition should be extremely maneuverable and flexible, so as to effectively enhance the individual ability of the athletes and better enhance the foundation of the group project. On the one hand, it is necessary to integrate with domestic and international development, improve the series of individual project settings, and ensure that the single-person project Health Qigong can be effectively integrated. On the other hand, it is necessary to demonstrate the ability of the routines and innovations by means of collective performance projects and group projects, reflecting the strength of the entire sports team, providing the audience with a very rich viewing effect and market value competition performance, so that the overall performance project can meet the needs of the martial arts routine market to enhance the audience's expectations.

\section{Strengthening Competition Model Innovation}

On the one hand, in the selection of the participating teams, in addition to the professional routine team and the major sports colleges, other participating teams can participate in the online registration form and pay the corresponding deposit to ensure that the team can improve the competition after entering the top 16 the comprehensiveness guarantees the level of competition. On the other hand, in a single item, each individual item can save 2 people to increase the number of players, promote the intensity of the game, and enhance participation and popularity. In the group project, each team is limited to 6 people, and finally 4 of the 5 people get the best score. Five people were reported in the finals, four people played, and 
three people took the best scores to minimize the uncontrollable factors. In addition, in the exhibition game, a team of about 20 people is allowed, and at least 4 women are allowed to participate in the free-form arrangement. The competition and performance sequence can be determined according to the lottery.

\section{Competition Method Innovation and Development}

The first is the content framework of the Health Qigong competition. During the qigong competition, each set of movements consists of 4 segmentation actions, each of which has 10 to 15 small movements. Each movement must ensure that it travels in the same direction, making full use of the site, ensuring difficult action links and improving overall operational integrity. The second is the basic action rules for sound action combinations. The various movements and hand types in Health Qigong must be effectively combined, and the specific actions must be determined. It is necessary to clarify the basic rules of action and self-selection actions, and deduct points for the lack of prescribed actions, and effectively add extra points for effective innovation of selfselected actions, and deduct points for violations of qigong development regular actions, thereby improving overall action specifications. The third is the difficulty of the action and the link provisions. In each different difficulty setting action, it is required to complete the key points of the action. For the completion of the basic score assessment, the connection score should also be effectively determined to ensure that the athlete can effectively play his or her own ability in the Health Qigong competition, and choose the appropriate according to their own characteristics. Exercises are drilled to improve the overall consistency of the action. The fourth is the provisions of the competition arrangement. In the specific competition arrangement, it is necessary to set up different venues in the preliminaries and finals to compete at the same time, adopt the elimination system to enter the finals, and adopt the circular style in the finals to determine the semi-finals and finalists for different sessions, and to perform exercises according to different combinations of actions. Promote the difficulty and aesthetic development of the routine. The fifth is to improve the requirements of the scoring rules. In the fitness qigong routine scoring rules, there is no upper limit to the method of breaking the score of the past. According to the method of gymnastics sports diving in the Olympic Games, the score is given according to the athlete's action combination difficulty coefficient, and a basic score is given. On this basis, detailed difficulty combination, connection score, innovation, completion and other aspects are determined to ensure the integrity of the scoring rules. During the game, VR photography and other assistive technologies should be added to ensure that the referees can clearly see the athletes' movements; the referee scores have real-time broadcasts to ensure fair and fair competition.

\section{CONCLUSION}

With the acceleration of the supply-side structural reform and the development of the internationalization path, the fitness Qigong routine competition system cannot meet the diverse needs of the masses in terms of project setting, content innovation, time and place. The reform of the Health Qigong Sports Competition System has a strong driving value for constructing the context of leisure sports culture and clarifying the entanglement of the traditional cultural system. Under the supply-side reform perspective, the location and time of the Health Qigong routine competition, the establishment of the competition system innovation project, the innovation and development of the competition method, and the competition model should be correspondingly innovated.

\section{REFERENCES}

[1] Song Ya, Mei Jiashun. Research on the development strategy of Health Qigong under the background of "One Belt, One Road" strategy [J]. Hubei Sports Science and Technology. 2017(09):56.

[2] Cui Yongsheng, Yang Huixin. Investigation and Analysis of the Characteristics of Health Qigong Practices [J]. Sports Culture Guide. 2017(06):31.

[3] Wang Yingwei, Xiang Hanping. Research on the "simpleness" of Health Qigong — Based on Chinese traditional aesthetics [J]. Journal of Wuhan Institute of Physical Education. 2017(09):12.

[4] Zhai Xianglin. Analysis and Discussion on Further Perfecting the Existing Health Qigong Competition Forms [J]. Zhonghua Wushu (Research). 2015(03):40. 